\title{
A REMARK ON THE INJECTIVITY OF THE SPECIALIZATION HOMOMORPHISM
}

\author{
IVica Gusić and Petra Tadić
}

University of Zagreb, Croatia

Abstract. Let

$$
E: y^{2}=\left(x-e_{1}\right)\left(x-e_{2}\right)\left(x-e_{3}\right)
$$

be a nonconstant elliptic curve over $\mathbb{Q}(T)$. We give sufficient conditions for a specialization homomorphism to be injective, based on the unique factorization in $\mathbb{Z}[T]$ and $\mathbb{Z}$.

The result is applied for calculating exactly the Mordell-Weil group of several elliptic curves over $\mathbb{Q}(T)$ coming from a paper by Rubin and Silverberg.

\section{INTRODUCTION}

Let $E=E(T)$ be a nonconstant elliptic curve over $\mathbb{Q}(T)$, i.e. an elliptic curve that is not isomorphic over $\mathbb{Q}(T)$ to an elliptic curve over $\mathbb{Q}$. By Silverman's specialization theorem ([6, Theorem III.11.4]), for all but finitely many $t \in \mathbb{Q}$, the specialization homomorphism

$$
E(\mathbb{Q}(T)) \rightarrow E(t)(\mathbb{Q})
$$

is injective, where $E(t)$ is the specialization of $E(T)$. Therefore the rank of $E(\mathbb{Q}(T))$ is finite and, by Mazur's theorem, the torsion group of $E(\mathbb{Q}(T))$ is one of the following groups:

$$
\mathbb{Z} / n \mathbb{Z}, 1 \leq n \leq 10 \text { or } n=12 \text {, or } \mathbb{Z} / 2 \mathbb{Z} \oplus \mathbb{Z} / 2 n \mathbb{Z}, 1 \leq n \leq 4 .
$$

Here we observe nonconstant elliptic curves $E$ over $\mathbb{Q}(T)$ given by an equation of the form

$$
E: y^{2}=\left(x-e_{1}\right)\left(x-e_{2}\right)\left(x-e_{3}\right), e_{1}, e_{2}, e_{3} \in \mathbb{Z}[T],
$$

2010 Mathematics Subject Classification. 11G05, 14H52.

Key words and phrases. Elliptic curve, specialization homomorphism, rank, generators. 
and give sufficient conditions on the coefficients of the curve (specifically $\left.e_{1}(T), e_{2}(T), e_{3}(T)\right)$ for a specialization homomorphism to be an injection. The details are in Section 3. Basically the factorization in the unique factorization domains $\mathbb{Z}[T]$ and $\mathbb{Z}$ plays a crucial role in the question of the injectivity of the specialization homomorphism.

The proof of this result uses the idea used in the paper by Dujella ([2, Theorem 4]), which relies on the homomorphism $\theta$ (see $[3,4.4]$ ).

The obtained result may lead to determining the rank and even proving that a certain set of points are free generators of an elliptic curve over $\mathbb{Q}(T)$ in the form (1.2), basically by looking at an elliptic curve over $\mathbb{Q}$ (one of its specialized curves which satisfies the condition of the Theorem 3.1).

In Section 4 we apply the result to a certain family of elliptic curves from the paper by Rubin and Silverberg ([5, Theorem 4.1]). For several concrete elliptic curves over $\mathbb{Q}(T)$, we calculate the rank and prove that a given set of points are free generators over $\mathbb{Q}(T)$. This is done by observing the curve's coefficients in $\mathbb{Q}(T)$ and in addition the rank and torsion and free generators of an elliptic curve over $\mathbb{Q}$ (one of its specializations). The key to this is the existence of efficient algorithms for finding free generators of a large class of elliptic curves over $\mathbb{Q}$, which is available through John Cremona's program mwrank ([1]).

\section{The Homomorphism $\theta$}

Let $K$ be the field of rational numbers $\mathbb{Q}$ or the field of rational functions $\mathbb{Q}(T)$ in the variable $T$ over $\mathbb{Q}$, let $R$ be the ring of integers $\mathbb{Z}$ or the ring $\mathbb{Z}[T]$ of polynomials in the variable $T$ over $\mathbb{Z}$, respectively. Thus, $R$ is a unique factorization domain.

Let us define the maps

$$
\theta_{i}^{K}: E(K) \rightarrow K^{\times} /\left(K^{\times}\right)^{2}, i=1,2,3
$$

by

$$
\begin{aligned}
\theta_{i}^{K}(x, y) & =x-e_{i}, \text { if } x \neq e_{i}, \\
\theta_{i}^{K}\left(e_{i}, 0\right) & =\left(e_{j}-e_{i}\right)\left(e_{k}-e_{i}\right), \text { where } i \neq j \neq k \neq i, \\
\theta_{i}^{K}(O) & =1 .
\end{aligned}
$$

Put $\theta^{K}:=\left(\theta_{1}^{K}, \theta_{2}^{K}, \theta_{3}^{K}\right)$. Note that $K^{\times} /\left(K^{\times}\right)^{2}$ has a natural structure of a multiplicative group. Then $\left(K^{\times} /\left(K^{\times}\right)^{2}\right)^{3}$ has the corresponding group structure of the direct product.

LEMMA 2.1. The map $\theta^{K}: E(K) \rightarrow\left(K^{\times} /\left(K^{\times}\right)^{2}\right)^{3}$ is a homomorphism of groups with the kernel $2 E(K)$. Thus, $\operatorname{Im}\left(\theta^{K}\right) \cong E(K) / 2 E(K)$.

Proof. The first part of the statement is in [3, Chapter 6, Proposition (4.3)]. The second part follows from [3, Chapter 1, Theorem (4.1)]. 
We restrict consideration to nonconstant elliptic curves $E$ over $K$ given by

$$
E: y^{2}=\left(x-e_{1}\right)\left(x-e_{2}\right)\left(x-e_{3}\right), e_{j} \in R .
$$

It is easy to see that $E(K) / 2 E(K)$ has $2^{\operatorname{rank}(E(K))+2}$ elements.

For each $P \in E(K)$ there exists exactly one triple

$$
\mu^{K}:=\left(\mu_{1}^{K}, \mu_{2}^{K}, \mu_{3}^{K}\right) \in\left(R^{\times}\right)^{3},
$$

where $\mu_{j}^{K}=\mu_{j}^{K}(P), j=1,2,3$, such that the following three conditions are satisfied

(i)

$$
\begin{aligned}
& \theta_{1}^{K}(P) \equiv \mu_{1}^{K} \mu_{2}^{K}\left(\bmod \left(K^{\times}\right)^{2}\right), \\
& \theta_{2}^{K}(P) \equiv \mu_{1}^{K} \mu_{3}^{K}\left(\bmod \left(K^{\times}\right)^{2}\right), \\
& \theta_{3}^{K}(P) \equiv \mu_{2}^{K} \mu_{3}^{K}\left(\bmod \left(K^{\times}\right)^{2}\right),
\end{aligned}
$$

(ii) $\mu_{j}^{K}$ are square-free and pairwise coprime in $R$, and

(iii) If $R=\mathbb{Z}[T]$ then the leading coefficient of $\mu_{1}^{\mathbb{Q}(T)} \in \mathbb{Z}[T]$ is positive, and if $R=\mathbb{Z}$ then $\mu_{1}^{\mathbb{Q}} \in \mathbb{Z}$ is positive.

REMARK 2.2. Note that since $e_{1}, e_{2}, e_{3} \in R$ we have

$$
\mu_{1}^{K}\left|e_{1}-e_{2}, \mu_{2}^{K}\right| e_{1}-e_{3}, \mu_{3}^{K} \mid e_{2}-e_{3} .
$$

These relations will be crucial in the proof of Theorem 3.1.

It is easy to see that

$$
\mu^{K}(P)=\mu^{K}(Q) \text { if and and only if } \theta^{K}(P)=\theta^{K}(Q) .
$$

Therefore, by Lemma 2.1,

$$
\mu^{K}(P)=\mu^{K}(Q) \text { if and only if } Q-P \in 2 E(K) .
$$

Especially,

$$
\mu^{K}(P)=(1,1,1) \text { if and only if } P \in 2 E(K) .
$$

We will be using $\theta$ and $\left(\mu_{1}, \mu_{2}, \mu_{3}\right)$ for $R=\mathbb{Z}[T]$ and $K=\mathbb{Q}(T)$.

\section{The INJECTIVITY OF THE SPECIALIZATION HOMOMORPHISM}

The main theorem in this section gives sufficient conditions on the coefficients of elliptic curves over $\mathbb{Q}(T)$ in the form (1.2), for a specialization homomorphism $T \mapsto t_{0}$ to be injective. Specifically, if the factors in the factorization of $\left(e_{1}(T)-e_{2}(T)\right) \cdot\left(e_{1}(T)-e_{3}(T)\right) \cdot\left(e_{2}(T)-e_{3}(T)\right)$ in $\mathbb{Z}[T]$ evaluated at $T=t_{0}$ have a certain property concerning its factorizations in $\mathbb{Z}$, then the injectivity of the specialization homomorphism $T \mapsto t_{0}$ can be concluded. 
Before the main theorem we mention the following. For a given non-zero rational number $q=\frac{a}{b},(a, b \in \mathbb{Z})$, let $\operatorname{core}(q)$ denote the integer square-free part of $q$, meaning the integer that is the the square-free part of $a \cdot b$. For example, the integer square-free part of $\frac{5}{12}$ is 15 . For a non-zero integer $m$ let $\operatorname{rad}(m)$ (called the radical of $m$ ) denote the product of all different prime divisors of $m$.

THEOREM 3.1. Let $t_{0} \in \mathbb{Q}$. Let $E$ be the nonconstant elliptic curve over $\mathbb{Q}(T)$, given by the equation

$$
E=E(T): y^{2}=\left(x-e_{1}\right)\left(x-e_{2}\right)\left(x-e_{3}\right),\left(e_{1}, e_{2}, e_{3} \in \mathbb{Z}[T]\right) .
$$

Factor

$$
\left(e_{1}-e_{2}\right) \cdot\left(e_{1}-e_{3}\right) \cdot\left(e_{2}-e_{3}\right)=a \cdot f_{1}^{a_{1}}(T) \cdots f_{k}^{a_{k}}(T),
$$

where $a \in \mathbb{Z}$ and $f_{i} \in \mathbb{Z}[T]$ irreducible (of positive degree) and $a_{i} \geq 1$. Assume that for each $i=1, \ldots, k$ the integer square-free part of each of $f_{i}\left(t_{0}\right)$ has at least one prime factor that doesn't appear in the integer square-free part of any of the other $f_{j}\left(t_{0}\right)(\forall j \neq i)$ and doesn't appear in the factorization of the radical of a. This condition includes the assumption that $f_{i}\left(t_{0}\right)$ is nonzero $(i=1, \ldots, k)$.

With the above notations the condition can be written as:

$$
\frac{\left|\operatorname{core}\left(f_{i}\left(t_{0}\right)\right)\right|}{\operatorname{rad}\left[\operatorname{gcd}\left(\operatorname{core}\left(f_{i}\left(t_{0}\right)\right), \operatorname{rad}(a)\right) \cdot \prod_{j=1, j \neq i}^{k} \operatorname{gcd}\left(\operatorname{core}\left(f_{i}\left(t_{0}\right)\right), \operatorname{core}\left(f_{j}\left(t_{0}\right)\right)\right)\right]}>1,
$$

for all $i=1, \ldots, k$. Then the specialization homomorphism $E(\mathbb{Q}(T)) \rightarrow$ $E\left(t_{0}\right)(\mathbb{Q})$ is injective.

Proof. Since $\left(e_{1}\left(t_{0}\right)-e_{2}\left(t_{0}\right)\right) \cdot\left(e_{1}\left(t_{0}\right)-e_{3}\left(t_{0}\right)\right) \cdot\left(e_{2}\left(t_{0}\right)-e_{3}\left(t_{0}\right)\right) \neq 0$, the specialization $E\left(t_{0}\right)$ of $E(T)$ is an elliptic curve.

Let $P \in E(\mathbb{Q}(T)) \backslash\{O\}$. Then the first coordinate of $P$ is of the form $\frac{p(T)}{q(T)^{2}}$ with $p(T), q(T) \in \mathbb{Z}[T]$ coprime. Therefore

$$
\left\{\begin{array}{l}
p(T)-e_{1}(T) q^{2}(T)=\mu_{1}^{\mathbb{Q}(T)}(P) \mu_{2}^{\mathbb{Q}(T)}(P) \square_{\mathbb{Z}[T]}, \\
p(T)-e_{2}(T) q^{2}(T)=\mu_{1}^{\mathbb{Q}(T)}(P) \mu_{3}^{\mathbb{Q}(T)}(P) \square_{\mathbb{Z}[T]}, \\
p(T)-e_{3}(T) q^{2}(T)=\mu_{2}^{\mathbb{Q}(T)}(P) \mu_{3}^{\mathbb{Q}(T)}(P) \square_{\mathbb{Z}[T]},
\end{array}\right.
$$

where $\square_{\mathbb{Z}[T]}$ denotes a square of an element of $\mathbb{Z}[T]$. Let

$$
\psi: E(\mathbb{Q}(T)) \rightarrow E\left(t_{0}\right)(\mathbb{Q})
$$

be the specialization homomorphism (note that $\psi$ is everywhere well-defined under the conditions of the theorem). Let $\bar{\mu}_{j}^{\mathbb{Q}(T)}(P), j=1,2,3$ denote the rational numbers obtained from $\mu_{j}^{\mathbb{Q}(T)}(P)$, by the specialization $T \mapsto t_{0}$.

- We first prove that $\psi(P)=O$ implies $P \in 2 E(\mathbb{Q}(T))$ : Let $P \in$ $E(\mathbb{Q}(T)) \backslash\{O\}$, then $\psi(P)=O$ implies $q\left(t_{0}\right)=0$ (while $p\left(t_{0}\right) \neq 0$ ). We mention that $P \neq\left(e_{i}(T), 0\right),(i=1,2,3)$, so we are in the first 
case in the definition of $\theta$ which we will use to prove the statement. Therefore

$$
\left\{\begin{array}{l}
p\left(t_{0}\right)=\bar{\mu}_{1}^{\mathbb{Q}(T)}(P) \bar{\mu}_{2}^{\mathbb{Q}(T)}(P) \square_{\mathbb{Q}}, \\
p\left(t_{0}\right)=\bar{\mu}_{1}^{\mathbb{Q}(T)}(P) \bar{\mu}_{3}^{\mathbb{Q}(T)}(P) \square_{\mathbb{Q}}, \\
p\left(t_{0}\right)=\bar{\mu}_{2}^{\mathbb{Q}(T)}(P) \bar{\mu}_{3}^{\mathbb{Q}(T)}(P) \square_{\mathbb{Q}},
\end{array}\right.
$$

where $\square_{\mathbb{Q}}$ denotes a square of a rational number. We claim that $\mu_{i}^{\mathbb{Q}(T)}(P) \in\{-1,1\}$, for each $i$. Assume, for example, that $\mu_{2}^{\mathbb{Q}(T)}(P) \notin$ $\{-1,1\}$. By multiplying the first two above relations, we get

$$
p\left(t_{0}\right)^{2}=\bar{\mu}_{2}^{\mathbb{Q}(T)}(P) \bar{\mu}_{3}^{\mathbb{Q}(T)}(P) \square_{\mathbb{Q}} .
$$

- Assume that at least one of $\mu_{2}^{\mathbb{Q}(T)}(P), \mu_{3}^{\mathbb{Q}(T)}(P)$ has a positive degree. Then from Remark 2.2, the fact that $\mu_{j}^{\mathbb{Q}(T)}(P)$ are square-free and mutually coprime, and the condition of the Theorem, we conclude that $\bar{\mu}_{2}^{\mathbb{Q}(T)}(P) \bar{\mu}_{3}^{\mathbb{Q}(T)}(P)$ is not a square in $\mathbb{Q}$. It is in contradiction with (3.1).

- Assume that both $\mu_{2}^{\mathbb{Q}(T)}(P), \mu_{3}^{\mathbb{Q}(T)}(P)$ are constants. Since they are square-free and coprime, and $\mu_{2}^{\mathbb{Q}(T)}(P) \notin\{-1,1\}$ we get a contradiction with (3.1).

Since we know that $\mu_{i}^{\mathbb{Q}(T)}(P) \in\{-1,1\}$, for each $i$ and using the fact that $\mu_{1}^{\mathbb{Q}(T)}(P)>0$, we easily conclude that $\mu_{i}^{\mathbb{Q}(T)}(P)=1$ for $i=1,2,3$. Now we see that $\theta^{\mathbb{Q}(T)}(P)=(1,1,1)$, hence by $(2.3)$ we have $P \in 2 E(\mathbb{Q}(T))$.

Since $\psi(O)=O$ and $O \in 2 E(\mathbb{Q}(T))$, we proved that $\psi(P)=O$ implies $P \in 2 E(\mathbb{Q}(T))$.

- Now we prove that $\psi(P) \in 2 \operatorname{Im} \psi$ if and only if $P \in 2 E(\mathbb{Q}(T))$ : if $\psi(P) \in 2 \operatorname{Im} \psi$, then $\psi(P)=2 \psi(Q)$ for some $Q \in E(\mathbb{Q}(T))$, then $\psi(P-2 Q)=O$, which implies, by the former, that $P-2 Q \in 2 E(\mathbb{Q}(T))$. So $P \in 2 E(\mathbb{Q}(T))$. The rest is obvious.

We thus conclude that

$$
E(\mathbb{Q}(T)) / 2 E(\mathbb{Q}(T)) \cong \operatorname{Im} \psi / 2 \operatorname{Im} \psi .
$$

Since $\psi$ is injective on the torsion part $[6$, p. 272-273, proof of Theorem III.11.4], and since a possible form of the torsion part is

$$
\mathbb{Z} / 2 \mathbb{Z} \oplus \mathbb{Z} / 2 n \mathbb{Z}, 1 \leq n \leq 4,
$$

by (3.2) we conclude

$$
2^{\operatorname{rank}(E(\mathbb{Q}(T))+2}=2^{\operatorname{rank}(\operatorname{Im}(\psi))+2},
$$

hence the rank of $E(\mathbb{Q}(T))$ is the same as the rank of $\operatorname{Im}(\psi)$.

Let $\bar{\psi}: E(\mathbb{Q}(T)) \otimes_{\mathbb{Z}} \mathbb{Q} \rightarrow \operatorname{Im} \psi \otimes_{\mathbb{Z}} \mathbb{Q}$ be the $\mathbb{Q}$-linear map corresponding to $\psi: E(\mathbb{Q}(T)) \rightarrow \operatorname{Im} \psi$. Since $\bar{\psi}$ is a surjective linear map among vector spaces 
of the same dimension, it is injective. By the fact that $\psi$ is injective on the torsion part, we conclude that $\psi$ is injective, too.

This result could be applied to determining the rank (and even the free generators) of an elliptic curves over $\mathbb{Q}(T)$ in the form (1.2), by basically choosing a good candidate $t_{0} \in \mathbb{Q}$ that of course satisfies the conditions of Theorem 3.1 and looking at an elliptic curve over $\mathbb{Q}$ (one of its specialized curves corresponding to $T=t_{0}$ ).

The following Corollary is used in the next section.

Corollary 3.2. Let $t_{0} \in \mathbb{Q}$. Let $E$ be the nonconstant elliptic curve over $\mathbb{Q}(T)$ in the form (1.2). If the condition from Theorem 3.1 is satisfied, and if

$$
\left|E(\mathbb{Q}(T))_{\text {Tors }}\right|=\left|E\left(t_{0}\right)(\mathbb{Q})_{\text {Tors }}\right|
$$

and there exist $P_{1}, \ldots, P_{r} \in E(\mathbb{Q}(T))$ such that $P_{1}\left(t_{0}\right), \ldots, P_{r}\left(t_{0}\right)$ are the free generators of $E\left(t_{0}\right)(\mathbb{Q})$, then the specialization homomorphism

$$
E(\mathbb{Q}(T)) \rightarrow E\left(t_{0}\right)(\mathbb{Q})
$$

is an isomorphism.

Thus $E(\mathbb{Q}(T))$ and $E\left(t_{0}\right)(\mathbb{Q})$ have the same rank $r$, and $P_{1}, \ldots, P_{r}$ are the free generators of $E(\mathbb{Q}(T))$.

PRoOF. The specialization is obviously an epimorphism, and by Theorem 3.1 it is an isomorphism.

REMARK 3.3. If $\left|E\left(t_{0}\right)(\mathbb{Q})_{\text {Tors }}\right|=4$, then the condition $\left|E(\mathbb{Q}(T))_{\text {Tors }}\right|=$ $\left|E\left(t_{0}\right)(\mathbb{Q})_{\text {Tors }}\right|$ is satisfied.

\section{Application to a family of Rubin and Silverberg}

Now we will give an example of the usage of the main Theorem 3.1 for obtaining new results concerning the paper by Rubin and Silverberg [5, Theorem 4.1]. We will determine the rank and free generators of several elliptic curves over $\mathbb{Q}(T)$ using Theorem 3.1 (moreover Corollary 3.2), by observing for each, its coefficients in $\mathbb{Z}[T]$ and one of its specialized curves over $\mathbb{Q}$. The possibility of determining the free generators of a large class of elliptic curve over $\mathbb{Q}$ is of essential importance for this, for which we use John Cremona's program mwrank ([1]).

The program mwrank uses 2-descent via 2-isogeny to determine the rank of an elliptic curve $E$ over $\mathbb{Q}$, and obtain a set of points which generate $E(\mathbb{Q})$ modulo $2 E(\mathbb{Q})$, and finally saturate it to a full basis over $\mathbb{Z}$ for $E(\mathbb{Q})$.

ExAmple 4.1. Let $a \in \mathbb{Q}^{\times}$, let $\lambda=-2 a^{2}$, and let $g^{(a)}(T)$ be the polynomial of degree 12 in $T$

$$
g^{(a)}(T)=2 N(\lambda, T)\left(N(\lambda, T)-2 D(\lambda, T)^{2}\right)\left(N(\lambda, T)-2 \lambda D(\lambda, T)^{2}\right),
$$


where

$$
\begin{aligned}
D(\lambda, T)= & \lambda(2 \lambda-1) T^{2}+2-\lambda \\
N(\lambda, T)= & \lambda^{2}(\lambda+1)(2 \lambda-1)^{2} T^{4}-4 \lambda^{2}(\lambda-1)(2 \lambda-1) T^{3} \\
& +2 \lambda(\lambda+1)\left(2 \lambda^{2}-3 \lambda+2\right) T^{2} \\
& -4 \lambda(\lambda-1)(\lambda-2) T+(\lambda-2)^{2}(\lambda+1) .
\end{aligned}
$$

In $[5$, Theorem 4.1$]$ it is proven that the elliptic curve $C^{(a)}$ over $\mathbb{Q}(T)$ with equation

$$
g^{(a)}(T) y^{2}=x(x-1)(x-\lambda)
$$

has rank at least 3 , with independent points $P^{(a)}, Q^{(a)}, R^{(a)} \in C^{(a)}(\mathbb{Q}(T))$ given by

$$
\begin{aligned}
P^{(a)}= & \left(\frac{N(\lambda, T)}{2 D(\lambda, T)^{2}}, \frac{1}{4 D(\lambda, T)^{3}}\right) \\
Q^{(a)}= & \left(\frac{\lambda^{2}\left(D(\lambda, T)^{2}-4 \lambda T(T-1)(\lambda(2 \lambda-1) T+2-\lambda)\right)}{\left(\lambda(2 \lambda-1) T^{2}-2 \lambda(2 \lambda-1) T+\lambda-2\right)^{2}},\right. \\
& \left.\frac{a \lambda}{\left(\lambda(2 \lambda-1) T^{2}-2 \lambda(2 \lambda-1) T+\lambda-2\right)^{3}}\right), \\
R^{(a)}= & \left(\frac{D(\lambda, T)^{2}+4 \lambda T(T-1)(\lambda(2 \lambda-1) T+2-\lambda)}{\lambda\left(\lambda(2 \lambda-1) T^{2}-(2 \lambda-4) T+\lambda-2\right)^{2}},\right. \\
& \left.-\frac{a}{\lambda^{2}\left(\lambda(2 \lambda-1) T^{2}-(2 \lambda-4) T+\lambda-2\right)^{3}}\right) .
\end{aligned}
$$

By [7, Section 4, Corollary 1] and [5, Remark 2.12], we know that the rank of $C^{(a)}$ over $\mathbb{Q}(T)$ is at most 5 , for each $a$. Now we will show that for each integer value $a$, where $1 \leq a \leq 60$, the elliptic curve $C^{(a)}$ over $\mathbb{Q}(T)$ has rank exactly equal to 3 and torsion $\mathbb{Z} / 2 \mathbb{Z} \oplus \mathbb{Z} / 2 \mathbb{Z}$, where free generators are the points

$$
P^{(a)}, Q^{(a)}, R^{(a)} \in C^{(a)}(\mathbb{Q}(T))
$$

given above. This strongly suggests that the rank in the family $C^{(a)}$ is constant and equals to 3 , as well as that $P^{(a)}, Q^{(a)}, R^{(a)}$ are free generators.

The coordinate transformation

$$
(x, y) \mapsto\left(g^{(a)}(T) \cdot x, g^{(a)}(T)^{2} \cdot y\right)
$$

applied to the elliptic curve $C^{(a)}$ over $\mathbb{Q}(T)$ leads to the elliptic curve over $\mathbb{Q}(T)$ given by the equation

$$
y^{2}=x\left(x-g^{(a)}(T)\right)\left(x-\lambda g^{(a)}(T)\right),
$$

which we also denote by $C^{(a)}$. The corresponding points also remain denoted as the old ones. Then

$$
e_{1}(T)=0, e_{2}(T)=g^{(a)}(T), e_{3}(T)=\lambda g^{(a)}(T),
$$


and four torsion points are $O,(0,0),\left(g^{(a)}(T), 0\right),\left(\lambda g^{(a)}(T), 0\right)$.

Proposition 4.2. Let $a$ be an integer such that $1 \leq a \leq 60$.

The elliptic curve $C^{(a)}$ over $\mathbb{Q}(T)$ has rank 3 , more precisely

$$
C^{(a)}(\mathbb{Q}(T)) \cong \mathbb{Z} / 2 \mathbb{Z} \oplus \mathbb{Z} / 2 \mathbb{Z} \oplus \mathbb{Z}^{3},
$$

and the points $P^{(a)}, Q^{(a)}, R^{(a)}$ are free generators of the group $C^{(a)}(\mathbb{Q}(T))$.

Proof. Note that $C^{(a)}$ is a nonconstant elliptic curve over $\mathbb{Q}(T)$ for each $a \neq 0$, although its $j$-invariant is a rational constant. Therefore, we can apply Theorem 3.1 and Corollary 3.2. First we will give a detailed proof for $a=1$

For $a=1$ and $t_{0}=4$ we have

- the elliptic curve $C^{(1)}(4)$ over $\mathbb{Q}$ is given by the equation

$y^{2}=x^{3}+502511523471360 x^{2}-505035662443014384369480499200 x$,

- the torsion group has four elements, mwrank ([1]) showed that $C^{(1)}(4)(\mathbb{Q})$ has rank 3 and free generators

$$
\begin{aligned}
G_{1} & =\left(-\frac{1689903343134720000}{1849},-\frac{863283322778865481285632000}{79507}\right), \\
G_{2} & =(790444733644800,20214846265347853516800), \\
G_{3} & =(13076929429218304,-1521697307273039513157632) .
\end{aligned}
$$

- using the commands elladd and ellsub in Pari ([4]) we obtain

$$
\begin{aligned}
& P^{(1)}(4)=\left(-2 g^{(1)}(4), 0\right)-G_{2}-G_{3}, \\
& Q^{(1)}(4)=(0,0)+G_{1}+G_{2}, \\
& R^{(1)}(4)=\left(-2 g^{(1)}(4), 0\right)+G_{2} .
\end{aligned}
$$

Thus we conclude that $P^{(1)}(4), Q^{(1)}(4), R^{(1)}(4)$ are free generators of the group $C^{(1)}(4)(\mathbb{Q})$ which has rank 3 .

- so we conclude that $\psi: C^{(1)}(\mathbb{Q}(T)) \rightarrow C^{(1)}(4)(\mathbb{Q})$ is a surjection.

- we have

$$
e_{1}(T)=0, e_{2}(T)=g^{(1)}(T), e_{3}(T)=-2 g^{(1)}(T),
$$

so

$$
\begin{aligned}
& \left(e_{1}(T)-e_{2}(T)\right) \cdot\left(e_{1}(T)-e_{3}(T)\right) \cdot\left(e_{2}(T)-e_{2}(T)\right) \\
& \quad=-9172942848 \cdot\left(25 T^{4}+60 T^{3}-16 T^{2}-24 T+4\right)^{3} \\
& \quad \cdot\left(25 T^{4}+20 T^{3}+8 T^{2}-8 T+4\right)^{3} \cdot\left(25 T^{4}-20 T^{3}+32 T^{2}+8 T+4\right)^{3},
\end{aligned}
$$


thus

$$
\begin{aligned}
\operatorname{rad}(a) & =6, \\
k & =3, \\
f_{1}(T) & =25 T^{4}+60 T^{3}-16 T^{2}-24 T+4, \\
f_{2}(T) & =25 T^{4}+20 T^{3}+8 T^{2}-8 T+4, \\
f_{3}(T) & =25 T^{4}-20 T^{3}+32 T^{2}+8 T+4 .
\end{aligned}
$$

If we take $t_{0}=4$ then we have the "prime" conditions of Theorem 3.1:

$$
\begin{aligned}
\operatorname{rad}(a) & =2 \cdot 3, \\
f_{1}(4) & =2^{2} \cdot 2473 \\
f_{2}(4) & =2^{2} \cdot 5 \cdot 389 \\
f_{3}(4) & =2^{2} \cdot 13 \cdot 109 .
\end{aligned}
$$

Thus the prime for $f_{1}(4)$ is 2473 , the prime for $f_{2}(4)$ is 5 or 389 , and the prime for $f_{3}(4)$ is 13 or 109 .

Thus we conclude by Corollary 3.2 applied to $a=1$ and $t_{0}=4$, that the specialization homomorphism $\psi: C^{(1)}(\mathbb{Q}(T)) \rightarrow C^{(1)}(4)(\mathbb{Q})$ is an isomorphism, so

$$
C^{(1)}(\mathbb{Q}(T)) \cong C^{(1)}(4)(\mathbb{Q}) \cong \mathbb{Z} / 2 \mathbb{Z} \oplus \mathbb{Z} / 2 \mathbb{Z} \oplus \mathbb{Z}^{3},
$$

and finally since $\psi\left(P^{(1)}\right), \psi\left(Q^{(1)}\right), \psi\left(R^{(1)}\right)$ are free generators of $C^{(1)}(4)(\mathbb{Q})$ we conclude that $P^{(1)}, Q^{(1)}, R^{(1)}$ are free generators of $C^{(1)}(\mathbb{Q}(T))$ which has rank 3.

The Table 4.1. below, shows for integer values $a \in\{1,2, \ldots, 60\}$, the corresponding $t_{0}$ for which the following conditions of the Corollary 3.2 are satisfied:

- the "prime" condition of Theorem 3.1 is satisfied for $e_{1}(T)=$ $0, e_{2}(T)=g^{(a)}(T), e_{3}(T)=\lambda g^{(a)}(T)$,

- the torsion subgroup of $C^{(a)}\left(t_{0}\right)(\mathbb{Q})$ has four elements,

- the rank of $C^{(a)}\left(t_{0}\right)(\mathbb{Q})$ is 3 , and free generators $G_{1}, G_{2}, G_{3}$ are found using mwrank ([1])

- the combination of $P^{(a)}\left(t_{0}\right), Q^{(a)}\left(t_{0}\right), R^{(a)}\left(t_{0}\right)$ of the torsion point and the generators $G_{1}, G_{2}, G_{3}$ is checked, which shows that

$$
P^{(a)}\left(t_{0}\right), Q^{(a)}\left(t_{0}\right), R^{(a)}\left(t_{0}\right)
$$

are also the generators of $C^{(a)}\left(t_{0}\right)(\mathbb{Q})$

By Corollary 3.2 we conclude that for all integer values $a \in\{1, \ldots, 60\}$ the specialization $\psi: C^{(a)}(\mathbb{Q}(T)) \rightarrow C^{(a)}\left(t_{0}\right)(\mathbb{Q})$ is an isomorphism, so

$$
C^{(a)}(\mathbb{Q}(T)) \cong C^{(a)}\left(t_{0}\right)(\mathbb{Q}) \cong \mathbb{Z} / 2 \mathbb{Z} \oplus \mathbb{Z} / 2 \mathbb{Z} \oplus \mathbb{Z}^{3}
$$

and $P^{(a)}, Q^{(a)}, R^{(a)}$ are free generators of $C^{(a)}(\mathbb{Q}(T))$. 


\begin{tabular}{||c||c|c|c|c|c|c|c|c|c|c||}
\hline \hline $\mathbf{a}$ & 1 & 2 & 3 & 4 & 5 & 6 & 7 & 8 & 9 & 10 \\
\hline $\mathbf{t}_{0}$ & 4 & $\frac{21}{2}$ & -9 & $-\frac{3}{20}$ & 5 & 6 & $\frac{2}{7}$ & $-\frac{3}{8}$ & -7 & $-\frac{5}{2}$ \\
\hline \hline $\mathbf{a}$ & 11 & 12 & 13 & 14 & 15 & 16 & 17 & 18 & 19 & 20 \\
\hline $\mathbf{t}_{0}$ & -9 & 25 & 15 & -10 & 25 & $\frac{3}{16}$ & -7 & $-\frac{9}{2}$ & -21 & -8 \\
\hline \hline $\mathbf{a}$ & 21 & 22 & 23 & 24 & 25 & 26 & 27 & 28 & 29 & 30 \\
\hline $\mathbf{t}_{0}$ & $\frac{25}{3}$ & -9 & -9 & -8 & -8 & -10 & -8 & -7 & 4 & -8 \\
\hline \hline $\mathbf{a}$ & 31 & 32 & 33 & 34 & 35 & 36 & 37 & 38 & 39 & 40 \\
\hline $\mathbf{t}_{0}$ & -10 & $-\frac{5}{32}$ & -10 & 61 & $\frac{7}{5}$ & -6 & 4 & $\frac{1}{2}$ & 4 & -3 \\
\hline \hline $\mathbf{a}$ & 41 & 42 & 43 & 44 & 45 & 46 & 47 & 48 & 49 & 50 \\
\hline $\mathbf{t}_{0}$ & $\frac{2}{41}$ & $-\frac{23}{2}$ & 30 & $\frac{6}{11}$ & -6 & -13 & $-\frac{9}{47}$ & $-\frac{11}{3}$ & 3 & $\frac{13}{2}$ \\
\hline \hline $\mathbf{a}$ & 51 & 52 & 53 & 54 & 55 & 56 & 57 & 58 & 59 & 60 \\
\hline $\mathbf{t}_{0}$ & $-\frac{7}{3}$ & 4 & $\frac{55}{7}$ & $\frac{11}{2}$ & $\frac{47}{2}$ & -6 & 13 & $-\frac{15}{2}$ & -5 & $\frac{25}{3}$ \\
\hline \hline
\end{tabular}

Table 4.1. List of values $a$ and corresponding $t_{0}$

For obtaining the table we observed $t_{0}$ that satisfy Corollary 3.2 such that the numerator is in absolute value $\leq 80$ and the denominator minimal. We looked at $t_{0}$ for which the root number of $C^{(a)}\left(t_{0}\right)$ is -1 and after that we let mwrank try to calculate the rank (and free generators).

\section{ACKNOWLEDGEMENTS.}

The authors would like to sincerely thank professor Andrej Dujella. This article would not have been possible without his kind support, help, suggestions and useful comments.

\section{REFERENCES}

[1] J. E. Cremona, Algorithms for Modular Elliptic Curves, Cambridge Univ. Press, 1997.

[2] A. Dujella, A parametric family of elliptic curves, Acta Arith. 94 (2000), 87-101.

[3] D. Husemöller, Elliptic Curves, Second Edition GTM 111, Springer, New York, 2004.

[4] Pari/GP, version 2.3.3, Bordeaux, 2008, http://pari.math.u-bordeaux.fr/.

[5] K. Rubin and A. Silverberg, Rank frequencies for quadratic twists of elliptic curves, Experiment. Math. 10 (2001), 559-569.

[6] J. H. Silverman, Advanced Topics in the Arithmetic of Elliptic Curves, GTM 151, Springer, Berlin, 1994.

[7] C. L. Stewart and J. Top, On ranks of twists of elliptic curves and power-free values of binary forms, J. Amer. Math. Soc. 8 (1995), 943-973. 
I. Gusić

Faculty of Chemical Engin. and Techn.

University of Zagreb

Marulićev trg 19, 10000 Zagreb

Croatia

E-mail: igusic@fkit.hr

P. Tadić

Geotechnical faculty

University of Zagreb

Hallerova aleja 7, 42000 Varaždin

Croatia

E-mail: petra.tadic.zg@gmail.com, ptadic@gfv.hr

Received: 14.1.2012.

Revised: 17.2.2012. 